
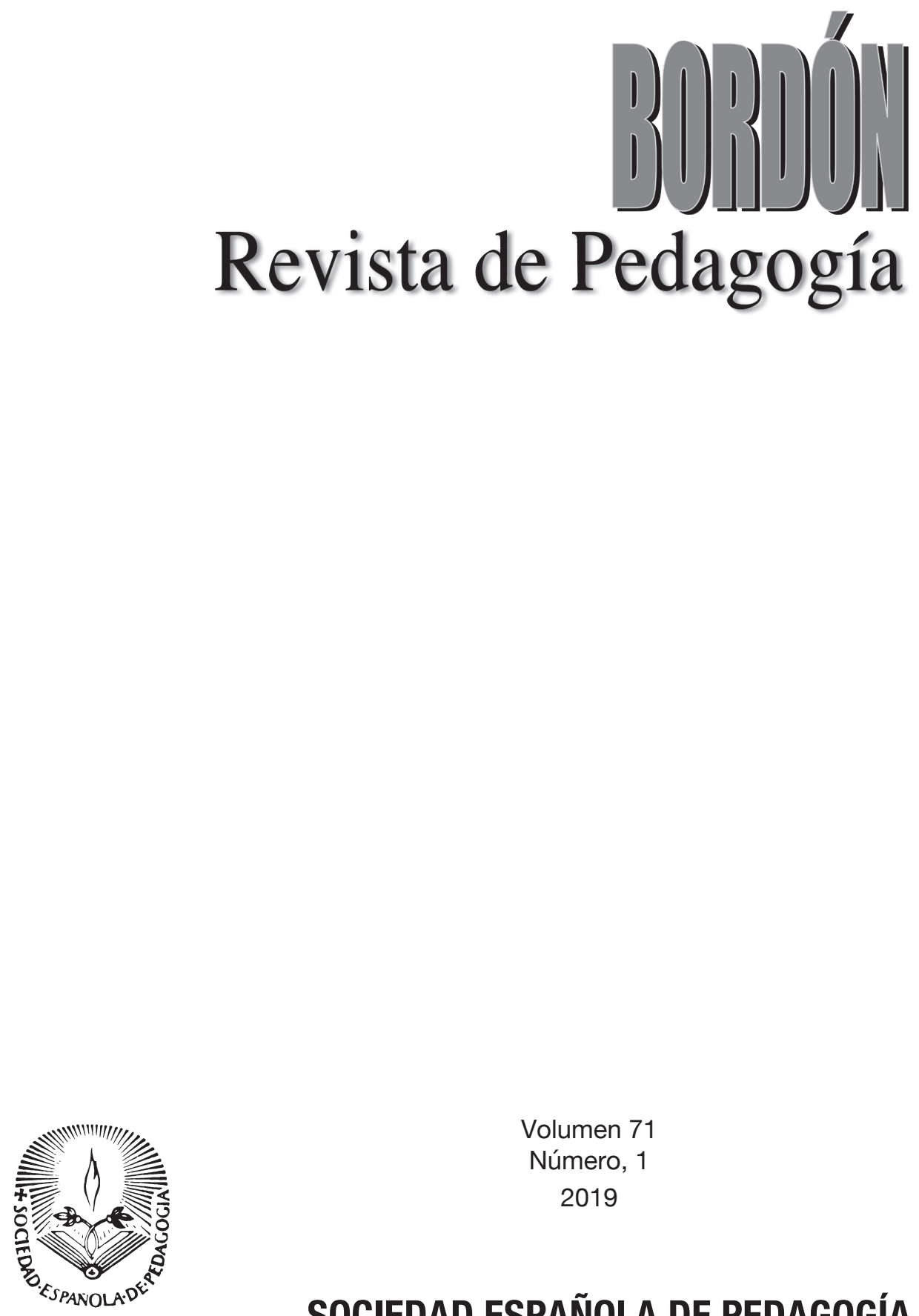

Volumen 71

Número, 1

2019 


\title{
LA COMPLEJA RELACIÓn DE LOS DOCEnTES CON LA LECTURA: EL COMPORTAMIEETOO LECTOR DEL PROFESORADO DE EDUCACIÓn INFAnTIL Y PRIMARIA EN FORMACIÓn
}

\author{
The complex relationship of teachers with reading as a skill: \\ the reading behavior of the teachers of Pre-school and Primary \\ Education in training
}

\author{
MARÍA ELCHE LARRAÑAGAY SANTIAGO YUBERO JIMÉNEZ
}

Universidad de Castilla-La Mancha

DOI: 10.13042/Bordon.2019.66083

Fecha de recepción: 14/06/2018 - Fecha de aceptación: 29/1I/2018

Autora de contacto / Corresponding Author: María Elche Larrañaga. E-mail: maria.elche@uclm.es

Fecha de publicación online: 23/01/2019

INTRODUCCIÓN. El interés de este trabajo es reflexionar, concretamente, sobre la calidad de la formación de los futuros maestros en el ámbito específico competencial como mediadores de lectura. Los últimos informes de los hábitos lectores de los alumnos españoles ponen en duda que haya existido evolución en esa materia, por lo que parece preciso hacer más efectiva la enseñanza de las primeras etapas educativas. Por otro lado, investigaciones previas han señalado la influencia que ejercen las experiencias lectoras de los maestros en su eficacia en este ámbito. Esta investigación está dirigida a estudiar el comportamiento y el compromiso lector de los estudiantes del Grado de Maestro para reflexionar sobre su desarrollo competencial respecto a la educación lectora de los niños y las niñas de Educación Infantil y Primaria. MÉTODO. En esta investigación participaron 861 estudiantes de las Universidades de Castilla-La Mancha y Santiago de Compostela, de los cursos primero a cuarto del Grado de Maestro en Educación Infantil (355) y Educación Primaria (506). Se evaluaron el comportamiento lector y el compromiso con la lectura. RESULTADOS. Los resultados señalaron relación entre comportamiento, compromiso y hábito lector. Conforme avanzan en la formación, se produce un aumento del hábito lector en los estudiantes del Grado de Maestro en Educación Primaria, pero no en los de Educación Infantil. Se evidenció la categoría de falsos lectores, así como un alto porcentaje de futuros maestros que parecían no reunir las condiciones personales precisas para desarrollar en sus alumnos un comportamiento lector voluntario. DISCUSIÓN. La universidad debe avanzar hacia una formación completa, sólida y coherente con las competencias que tienen desarrollar estos futuros profesionales como mediadores de lectura, potenciando su hábito lector en lo que se refiere tanto al comportamiento como al compromiso lector.

Palabras clave: Hábitos lectores, Intereses lectores, Maestros, Profesores lectores, Universidad. 


\section{Introducción}

Tras la completa incorporación del Espacio Europeo de Educación Superior (EEES) parece el momento de llevar a cabo un proceso de reflexión y revisión de la calidad de la formación que ofrece la universidad española. Este proceso ya se está produciendo en grados como Pedagogía (Torres y Vallejo, 2018); nuestro interés es llevarlo a cabo en los futuros maestros en el ámbito específico competencial como mediadores de lectura.

El último informe del Programme for International Student Assessment (PISA) (Organización para la Cooperación y el Desarrollo Económicos [OCDE], 2016) ofrece datos globales de lectura. España ha obtenido una puntuación de 496 puntos, siete por encima del informe de 2012. Los datos de España parecen reflejar un estancamiento desde el inicio de la prueba y, tal y como se afirma en este último informe, quizá los gobiernos deberían buscar maneras de hacer más efectiva la enseñanza. Aunque es difícil establecer razones concretas en la mejora de un sistema educativo, hay consenso en que una de las claves es mejorar la profesión docente. La Comisión Europea (2008) ya destacó la calidad del personal docente como uno de los principales factores que determinan el éxito académico de los estudiantes.

\section{¿Qué se espera de los maestros respecto} a la lectura?

Según la Orden ECI/3960/2007, de 19 de diciembre, por la que se establece el currículo y se regula la ordenación de la Educación Infantil, la adquisición de las competencias básicas se plantea como puente para el desarrollo integral de la persona. Entre estas, se concede especial importancia al desarrollo de las habilidades comunicativas en los diferentes lenguajes y formas de expresión, incluido el descubrimiento del placer de la lectura a través de la literatura infantil. Según el Real Decreto 126/2014, de 28 de febrero, por el que se establece el currículo básico de la Educación Primaria, la finalidad es garantizar una formación integral que contribuya al pleno desarrollo de la personalidad de los alumnos y de prepararlos para cursar los siguientes niveles. Entre las competencias del currículo que tienen que desarrollar en esta etapa se potencia el desarrollo de la comunicación lingüística y se hace hincapié en la comprensión lectora.

Los alumnos llegan a la escuela con una formación prelectora que han ido adquiriendo en sus experiencias en casa y en el entorno inmediato, a través de las interacciones sociales. El papel de los maestros en las etapas de Educación Infantil y Educación Primaria será ampliar esas competencias. Por ello, sin duda, los maestros deben tener conocimientos literarios actualizados y saber qué intereses concretos se adecúan a las necesidades de los alumnos, pero también deben ser capaces de plantear actividades de animación que realmente sean efectivas en la búsqueda del hábito lector y la familiarización con la literatura (López-Valero, Encabo y Jerez, 2016). Investigaciones previas han destacado el valor del contexto educativo para potenciar la lectura, sin dejar de lado el rol fundamental que desempeña el contexto familiar.

\section{¿Qué apoyo ofrece la universidad} a la formación lectora de los maestros?

Los datos del Estudio Internacional de Progreso en Comprensión Lectora (PIRLS) de 2006 sugieren que existe relación entre la relevancia que se da a la lectura durante la formación inicial del profesorado y la eficacia de los futuros docentes en este ámbito (Ministerio de Educación, Cultura y Deporte [MECD], 2007). Si accedemos a las páginas webs de las universidades, encontramos los documentos de grado de las universidades participantes en el estudio, en los que se especifican las distintas asignaturas que se imparten en el Grado de Maestro en Educación Infantil y Primaria. Analizándolas, observamos en ambas 
universidades materias troncales de lenguaje y literatura a lo largo de toda la titulación, como "Didáctica de la lengua y la literatura española", "Lingüística" y "Adquisición y desarrollo del lenguaje". Solo aparece en el Grado de Maestro en Educación Primaria de la Universidad de Castilla-La Mancha (UCLM) una asignatura de "Animación a la lectura", y la de "Intervención educativa en las dificultades del lenguaje oral y escrito" en el Grado de Maestro en Educación Primaria de la Universidad de Santiago de Compostela (USC), ambas con carácter optativo y solo ofertadas para alguna especialidad concreta (tabla 1).

También encontramos información detallada sobre las competencias que deben adquirir los estudiantes en estas titulaciones. En las competencias generales establecidas por la Orden ECI/3857/2007, de 27 de diciembre, por la que se establecen los requisitos para la verificación de los títulos universitarios oficiales, en las referidas al Grado de Maestro en Educación Primaria queda reflejada la competencia de "fomentar la lectura". Sin embargo, en las correspondientes al Grado de Maestro en Educación Infantil no hay ninguna referida a la lectura; aparece solamente la competencia de "expresarse oralmente y por escrito". En las competencias propias de la universidad no se hace referencia al fomento de la lectura como competencia de los graduados en maestro en ninguna de las dos universidades.

\section{Maestros y lectura}

Contamos cada vez con más evidencias sobre la influencia que ejercen los hábitos lectores de los maestros sobre sus prácticas docentes y en el fomento de la lectura en el aula, en relación con el modelamiento de actitudes y hábitos lectores de niños y niñas (Granado, 2014; Granado y Puig, 2014). Según distintos autores (Boggs y Golden, 2009; Trent, 2011), las experiencias personales de lectura de los futuros maestros ejercen una influencia decisiva sobre la formación en este campo. Otras investigaciones revelan cómo las propias prácticas lectoras de los maestros son trasladadas al aula (Cremin, Mottram, Collins, Powell y Safford, 2009; Granado y Puig, 2015). Applegate y Applegate (2004) afirman que un maestro que mantiene una relación débil con la lectura no puede contribuir a desarrollar en sus alumnos una motivación intrínseca por la práctica lectora. Esto podría indicar que el uso eficiente de prácticas de lectura que favorecen la experiencia del placer lector requiere un modelo de relación estrecha, intensa y vital con la lectura. Granado, Puig y Romero (2012) señalan, en la misma línea, que son los estudiantes del Grado de Maestro en Educación con mayor hábito lector quienes más importancia dan a los contenidos formativos relacionados con la promoción de la lectura frente a los de menor hábito, que circunscriben su interés a la formación centrada en la instrucción lectoescritora.

TABLA 1. Universidades y asignaturas analizadas en el estudio

UCLM (Cuenca)

USC

\begin{tabular}{|c|c|c|}
\hline \multirow{3}{*}{$\begin{array}{l}\text { Grado de } \\
\text { Maestro en } \\
\text { Educación } \\
\text { Infantil }\end{array}$} & Adquisición y desarrollo del lenguaje & Aprendizaxe da Lingua Oral \\
\hline & Didáctica de la lengua en educación infantil & Iniciación á Lectura e á Escritura \\
\hline & Literatura infantil y animación a la lectura & Literatura infantil e Dramatización \\
\hline \multirow{3}{*}{$\begin{array}{l}\text { Grado de } \\
\text { Maestro en } \\
\text { Educación } \\
\text { Primaria }\end{array}$} & Lingüística aplicada a la enseñanza & $\begin{array}{l}\text { Ensino e Aprendizaxe de Linguas en contextos } \\
\text { Multilingües }\end{array}$ \\
\hline & Didáctica de la literatura española & $\begin{array}{l}\text { Ensino e Apendizaxe de Competencias } \\
\text { Comunicativas: Lingua e Literatura }\end{array}$ \\
\hline & Didáctica de la lengua española & Literatura, Lectura e Creatividade \\
\hline
\end{tabular}


Como afirman De-Naeghel et al. (2014), el compromiso lector ha tomado un rol prominente sobre la lectura. El compromiso lector incluye la dedicación a la lectura, es decir, el tiempo dedicado a la lectura (Guthrie, Wigfield, Metsala y Cox, 1999) y los procesos motivacionales (Guthrie y Wigfield, 2000). Investigaciones previas han indicado que el compromiso lector correlaciona con el rendimiento lector (Guthrie, Klauda y Ho, 2013) y con la comprensión lectora (Wigfield et al., 2008). Los resultados en PISA indican que el compromiso con la lectura y el progreso lector se refuerzan mutuamente (Valenzuela, Gómez y Sotomayor, 2015). Esperamos que el hábito lector también se relacione con el compromiso lector.

\section{Hábitos de lectura de los futuros maestros}

No podemos olvidar que los jóvenes universitarios que cursan los estudios de Maestro en Educación Infantil y Primaria serán los primeros receptores, junto a padres y bibliotecarios, de la Literatura Infantil y Juvenil (LIJ). Como indica Cerrillo (2016), una de las características propias de la LIJ es la presencia de un adulto que facilite el contacto con los libros y acompañe la lectura cuando sea necesario. Ante tal responsabilidad, cabría esperar de los futuros maestros que tuvieran un amplio bagaje literario, un perfil de lectores competentes y una fuerte conciencia del compromiso que habrán de asumir.

Sin embargo, los últimos estudios nacionales e internacionales sobre los hábitos lectores de los estudiantes de Magisterio muestran que en su mayoría no son lectores habituales (Colomer y Munita, 2013; Díaz-Plaja y Prats, 2013; Granado, 2014; Granado et al., 2012; Sanjuán-Álvarez, 2013). Felipe (2016) encuentra que un 18,2\% de los 950 futuros maestros de Málaga admiten no haber leído un solo libro de forma voluntaria en todo el verano. Una investigación en la que participan 2175 estudiantes de doce universidades españolas (Larrañaga et al., 2008) determina que los estudiantes del Grado en Maestro leen una media de 4,6 libros al año, mucho menor a la media de los estudiantes universitarios; solo un $7,7 \%$ de los futuros maestros encuestados son lectores habituales y cuatro de cada diez son no lectores o falsos lectores. Los autores definen la categoría de falso lector por la influencia de la deseabilidad social, provocada por la imagen social positiva del lector, que lleva a querer generar una imagen de lector, aunque realmente no se lleve a cabo una conducta lectora habitual. En un estudio posterior, con 1116 estudiantes de doce universidades portuguesas (Yubero, Larrañaga y Pires, 2014), encuentran que el 10\% de la muestra de futuros maestros son no lectores; el $27,7 \%$, falsos lectores, y el 11,7\%, lectores habituales; el $47 \%$ informa que lee porque le gusta. Applegate y Applegate (2004) hallan en un estudio piloto realizado con 195 estudiantes estadounidenses de diversos programas de formación del profesorado de educación primaria que, en general, la mitad de los futuros maestros no están intrínsecamente motivados hacia la lectura. En un trabajo posterior (Applegate y Applegate, 2014), solamente la mitad de los 348 maestros en formación inicial que toman parte en el estudio se consideran lectores entusiastas. Del mismo modo, Felipe (2016) halla que el 52,9\% de los futuros maestros de Málaga de su estudio son lectores entusiastas, frente a un $47,2 \%$ de lectores no entusiastas.

Aunque PISA no mide la relación causal, informa de que los bajos niveles de rendimiento en lectura tienden a estar unidos a un bajo nivel de implicación y compromiso con la lectura (Valenzuela, Gómez y Sotomayor, 2015). En este sentido, si actuamos sobre los niveles de implicación y de compromiso con la lectura, podremos esperar que esto se relacione con un incremento de los niveles de rendimiento lector y, así, poder mejorar los hábitos lectores.

Por todo ello, destacamos la importancia de reflexionar sobre los maestros como lectores y detectar sus posibles necesidades para poder intervenir desde la formación universitaria en la tarea de los futuros maestros en relación con el fomento de la lectura. 
Tomando en consideración la relevancia del compromiso lector, el presente estudio tiene como objetivo analizar la relación del compromiso lector con el hábito lector. Por otra parte, atendiendo al rol de los maestros como mediadores lectores, el segundo objetivo es analizar el comportamiento y el compromiso lector de los estudiantes de los grados de Maestro en Educación Infantil y Educación Primaria para conocer su evolución durante la formación universitaria.

La primera hipótesis de estudio es que el compromiso lector estará relacionado con el hábito lector. En relación con la formación universitaria, esperamos que los estudiantes del Grado de Maestro en Educación Primaria, al estar incluidas en sus competencias el fomento de la lectura, incrementen el hábito lector y que, por tanto, se produzca un aumento tanto en el comportamiento lector como en el compromiso con la lectura desde el primer curso hasta su salida de la universidad.

\section{Método}

\section{Participantes}

En esta investigación participaron 861 estudiantes universitarios de primero a cuarto del Grado de Maestro en Educación Infantil (355) y Educación Primaria (506) de las diferentes facultades de la UCLM y de la USC, lo que supone casi un 25\% de los casos. Ambas universidades ofrecían los grados de Maestro en todos los campus. El conjunto de los grados de Maestro en Educación Infantil y Primaria que formaron parte de la investigación tenía un número aproximado de 3500 estudiantes. Para mantener un 95\% de confianza en el estudio, eran necesarios 306 participantes. Un 79,8\% (687) eran mujeres. La muestra comprendía alumnos de entre 18 y 51 años, siendo la edad media de 21,37 $(D T=3,61)$. En la tabla 2 se presenta la distribución de los estudiantes que participaron en el estudio.

Tabla 2. Distribución de los participantes en el estudio

Educación Infantil

n (\%)
Educación Primaria n (\%)

\begin{tabular}{lcc} 
Universidad & & $298(58,9)$ \\
\hline Castilla-La Mancha & $194(54,6)$ & $208(41,1)$ \\
\hline Santiago de Compostela & $161(45,4)$ & $138(27,3)$ \\
\hline Sexo & $35(9,6)$ & $368(72,7)$ \\
\hline Hombre & $320(90,4)$ & $438(85,3)$ \\
\hline Mujer & & $68(14,7)$ \\
\hline Edad & $287(81,3)$ & 21,15 \\
\hline $18-23$ & $68(18,7)$ & 3,58 \\
\hline Más de 23 & 21,70 & $165(32,6)$ \\
\hline Media & 3,65 & $145(28,7)$ \\
\hline Desviación típica & $73(20,6)$ & $152(30,0)$ \\
\hline Curso & $196(55,2)$ & $44(8,7)$
\end{tabular}




\section{Instrumento}

Para este estudio se empleó un cuestionario que recogía información sobre el comportamiento lector de los estudiantes universitarios, incluyendo información sobre la implicación y el compromiso con la lectura. Se les preguntó acerca de: tiempo dedicado a la lectura, número de libros leídos, percepción del nivel lector, motivación lectora (Larrañaga et al., 2008); gusto por la lectura, tiempo dedicado a la lectura en tiempo de ocio (Yubero et al., 2014); periodo en que leen voluntariamente (Encuesta de hábitos y prácticas culturales, Instituto Nacional de Estadística [INE], 2015); tipología lectora y perfil lector (Granado et al., 2012). También se evaluó el perfil lector con el que se identificaban en el momento de completar el cuestionario y antes de acceder a la universidad. En la tabla 3 se muestra la descripción de los ítems del instrumento utilizado.

\section{Procedimiento}

El muestreo fue intencional. Dentro de cada una de las universidades, el muestreo se realizó por conglomerados, tomando el aula como unidad muestral en función de la disponibilidad de los profesores y de los alumnos.

El cuestionario se pasó en las aulas de clase durante el segundo semestre del curso 2016/2017, previa autorización de los profesores colaboradores y en los espacios que ellos indicaron. Se explicaron la finalidad y las condiciones de estudio. La participación fue voluntaria y anónima y se aseguró la confidencialidad.

En todo momento velamos por cumplir con los requisitos marcados por el Código de Buenas Prácticas Científicas aprobado por el CSIC, en marzo de 2010.

Tabla 3. Descripción del instrumento

\begin{tabular}{|c|c|c|c|}
\hline Variable & Naturaleza & Descripción & Respuesta \\
\hline Hábito lector & Objetiva & $\begin{array}{l}\text { Frecuencia lectura } \\
\text { Número libros leídos }\end{array}$ & Categorizada \\
\hline Hábito lector & Subjetiva & $\begin{array}{l}\text { Perfil lector previo } \\
\text { Perfil lector actual }\end{array}$ & Categorizada \\
\hline Comportamiento & Objetiva & $\begin{array}{l}\text { Tipología lectora: } \\
\text { Revistas } \\
\text { Cómics } \\
\text { Ficción } \\
\text { No ficción } \\
\text { Prensa } \\
\end{array}$ & Escala tipo Likert \\
\hline Comportamiento lector & Objetiva & Estar leyendo & Dicotómica \\
\hline Comportamiento lector & Subjetiva & Nivel lector & Escala tipo Likert \\
\hline Compromiso lectura & Objetiva & Gusto lector & Escala 1 a 10 \\
\hline Compromiso lectura & Objetiva & $\begin{array}{l}\text { Motivación: } \\
\text { Me divierte } \\
\text { Me gusta } \\
\text { Me evado } \\
\text { Me informo } \\
\text { Aprendo } \\
\text { Estoy al día }\end{array}$ & Selección múltiple \\
\hline Compromiso lectura & Objetiva & Leer en tiempo de ocio & Escala tipo Likert \\
\hline Compromiso lectura & Objetiva & Periodo de lectura & Escala tipo Likert \\
\hline
\end{tabular}




\section{Análisis de datos}

En primer lugar, para evaluar el hábito lector, se seleccionaron dos preguntas de lectura voluntaria: la frecuencia de lectura y el número de libros leídos por placer durante el último año. Esta medida ya había sido utilizada en investigaciones previas sobre el análisis del comportamiento lector en estudiantes universitarios (Larrañaga y Yubero, 2005; Yubero et al., 2014; Yubero y Larrañaga, 2015), y se había informado de que en los resultados del cruce de respuestas entre ambas variables aparecían resultados inconsistentes. Se encontraban sujetos que informaban de comportamiento lector en una variable y de comportamiento no lector en la otra; parecía que algunos estudiantes querían ofrecer una imagen diferente a la real. Cruzamos ambas variables para construir el hábito lector. Solo consideramos "lector habitual" a los sujetos que responden en esta categoría en las dos variables y "lector ocasional" a los sujetos en los que se produce el cruce de dos valoraciones de lector ocasional y el de lector habitual con lector ocasional. Entra en la categoría de "no lector" el cruce de no lector en ambas categorías. La combinación de "no lector" en cualquiera de las dos variables con una valoración de lector (ocasional o habitual) en la otra, lo designamos como sujeto "falso lector", por entender que realmente es no lector, pero está queriendo dar una imagen lectora. De esta manera, obtuvimos cuatro grupos de lectores:

- No lectores: no leían nunca o casi nunca. Entre 0 y 2 libros al año.

- Lectores ocasionales: leían alguna vez al trimestre o al mes. Entre 3 y 10 libros al año.

- Lectores frecuentes: leían casi todos los días o más de una vez a la semana. Más de 10 libros al año.

- Falsos lectores: aquellos que en una variable se mostraban como lectores y en la otra como no lectores.

Para someter a prueba la primera hipótesis del estudio se realizó un análisis de regresión lineal por pasos, introduciendo en el primer paso las variables de comportamiento lector y en el segundo paso las variables de compromiso lector. Para someter a prueba la influencia de la formación universitaria, se realizaron contrastes estadísticos a través de chi-cuadrado sobre los valores del hábito lector, según el curso y sobre la percepción del perfil lector antes de entrar en la universidad y en el momento de completar el cuestionario. Con posterioridad, se realizaron análisis multivariantes para considerar conjuntamente la influencia del grado y el curso en el que se encuentra el estudiante sobre el comportamiento y el compromiso lector.

\section{Resultados}

El comportamiento lector explicó el 47\% de la varianza del hábito lector, el compromiso con la lectura añadía un 10\% de varianza. Los resultados de las regresiones (tabla 4) indicaron la influencia del comportamiento lector centrada en la lectura de ficción y no ficción y estar leyendo un libro en el momento de completar el cuestionario. También la influencia del compromiso lector sobre el hábito lector que aparecía en el gusto por la lectura, la motivación intrínseca, dedicar tiempo libre a la lectura y la regularidad en el periodo de lectura.

Para conocer si se producía alguna modificación del comportamiento lector por la decisión de convertirse en maestros, comparamos el perfil lector al que se adscribían como futuros maestros y el que percibían antes de iniciar los estudios de grado. Como puede observarse en la tabla 5, la mayoría de los futuros educadores se situaban en las categorías de lectura ocasional (lector ocasional y lector de vacaciones), casi el 25\% en las categorías de lectura frecuente (lector habitual y lector empedernido). No se encontraron diferencias significativas entre los estudiantes del Grado de Maestro en Educación Infantil y Educación Primaria. En la comparación entre el perfil actual y el 
perfil antes de acceder a los estudios universitarios la diferencia fue significativa en los dos grados de futuros maestros, $p<, 001$. Se reducía casi a la mitad el porcentaje de no lectores y se incrementaba ligeramente el porcentaje de lector empedernido.
También comparamos los porcentajes en las categorías de hábito lector según curso y grado (tabla 6). Observamos una reducción en el porcentaje de no lectores, pero el contraste solamente alcanzó significación para los estudiantes del Grado de Maestro en Educación Primaria.

TABLa 4. Regresión lineal del comportamiento lector y el compromiso con la lectura sobre el hábito lector

\begin{tabular}{|c|c|c|c|c|}
\hline & \multicolumn{2}{|c|}{ Modelo I } & \multicolumn{2}{|c|}{ Modelo II } \\
\hline & $\beta$ & $t$ & $\beta$ & $t$ \\
\hline \multicolumn{5}{|l|}{ Comportamiento lector } \\
\hline Revistas & 07 & $2,40^{*}$ &, 05 & 1,82 \\
\hline Cómic &, 06 & $2,25^{*}$ &, 03 & 1,42 \\
\hline Ficción & 41 & $13,72 * * *$ &, 18 & $5,42 * * *$ \\
\hline No ficción & , 16 & $5,86^{* * *}$ & ,08 & $3,32 * * *$ \\
\hline Prensa &, 06 & $2,24^{*}$ &, 05 & 1,83 \\
\hline Nivel lector & ,16 & $5,70 * * *$ &, 05 & 1,89 \\
\hline Estar leyendo &, 22 & $7,68 * * *$ &, 13 & $4,79 * * *$ \\
\hline \multicolumn{5}{|l|}{ Compromiso lector } \\
\hline Gusto lector & & &, 16 & $4,05^{* * *}$ \\
\hline Motivación intrínseca & & & 07 & $2,35^{*}$ \\
\hline Motivación extrínseca & & &,- 02 & $-0,73$ \\
\hline Leer tiempo ocio & & &, 29 & $8,88^{* * *}$ \\
\hline Periodo de lectura & & &, 11 & $3,97 * * *$ \\
\hline$R^{2}$ & \multirow{2}{*}{\multicolumn{2}{|c|}{$\begin{array}{c}, 47 \\
101,28 * * *\end{array}$}} & \multirow{2}{*}{\multicolumn{2}{|c|}{$\begin{array}{c}, 57 \\
89,78^{* * *}\end{array}$}} \\
\hline$F$ & & & & \\
\hline
\end{tabular}

Nota: ${ }^{*} p<, 05 ;{ }^{* *} p<, 01 ; p<, 001$.

Tabla 5. Perfil lector antes-después de acceder a la universidad, en porcentajes Total Infantil Primaria $\chi^{2}$ $p(w)$

Actual

\begin{tabular}{|c|c|c|c|c|c|}
\hline No leo & 6,3 & 5,6 & 6,8 & \multirow{5}{*}{1,14} & \multirow{5}{*}{$\begin{array}{r}, 887 \\
(, 02)\end{array}$} \\
\hline Lector ocasional & 40,4 & 40,4 & 40,4 & & \\
\hline Lector de vacaciones & 28,9 & 28,2 & 29,4 & & \\
\hline Lector habitual & 21,7 & 23,2 & 20,7 & & \\
\hline Lector empedernido & 2,7 & 2,5 & 2,8 & & \\
\hline \multicolumn{6}{|c|}{ Antes de acceder a la universidad } \\
\hline No leo & 12,4 & 10,7 & 13,5 & \multirow{5}{*}{3,75} & \multirow{5}{*}{$\begin{array}{r}, 441 \\
(, 06)\end{array}$} \\
\hline Lector ocasional & 38,5 & 37,5 & 39,2 & & \\
\hline Lector de vacaciones & 21,6 & 21,1 & 21,9 & & \\
\hline Lector habitual & 22,0 & 24,8 & 20,1 & & \\
\hline Lector empedernido & 5,6 & 5,9 & 5,4 & & \\
\hline$\chi^{2}$ & 736,16 & 252,71 & 503,46 & & \\
\hline$p(w)$ &, $000(, 59)$ &, $000(, 52)$ &, $000(, 65)$ & & \\
\hline
\end{tabular}


TABLA 6. Hábito lector según curso y grado, en porcentajes

\begin{tabular}{|c|c|c|c|c|c|c|c|c|c|c|c|c|}
\hline & & & tal & & & & ntil & & & Prir & aria & \\
\hline & $1^{\circ}$ & $2^{\circ}$ & $3^{\circ}$ & $4^{\circ}$ & $1^{\circ}$ & $2^{\circ}$ & $3^{\circ}$ & $4^{\circ}$ & $1^{\circ}$ & $2^{\circ}$ & $3^{\circ}$ & $4^{\circ}$ \\
\hline No leo & 29,8 & 24,6 & 17,2 & 15,4 & 21,9 & 28,1 & 18,8 & 14,3 & 33,3 & 20,0 & 16,6 & 15,9 \\
\hline Falso lector & 22,3 & 27,0 & 30,2 & 30,8 & 23,3 & 27,6 & 28,1 & 23,8 & 21,8 & 26,2 & 31,1 & 34,1 \\
\hline Lector ocasional & 41,2 & 39,2 & 45,1 & 44,6 & 47,9 & 35,7 & 37,5 & 57,1 & 38,2 & 44,1 & 48,3 & 38,6 \\
\hline Lector habitual & 6,7 & 9,1 & 7,4 & 9,2 & 6,8 & 8,7 & 15,6 & 4,8 & 6,7 & 9,7 & 4,0 & 11,4 \\
\hline$X^{2}$ & & & & & & & 95 & & & & & \\
\hline$p(w)$ & & & & & & & & & & ,003 & $(, 16)$ & \\
\hline
\end{tabular}

En el análisis multivariado de varianza (MANOVA) se realizó utilizando como variables independientes el grado y el curso. El modelo resultó significativo para el comportamiento lector [Lambda $\left.(6,805)=0,53, F=2409,91, p<, 001, \eta^{2}=, 95\right]$ y para el compromiso con la lectura [Lambda $(5,827)=$ $\left.0,90, F=1680,12 p<, 001, \eta^{2}=, 91\right]$.

En el análisis del comportamiento lector, la interacción del curso y el grado resultó significativa $[$ Lambda $(18,2277)=0,96, F=1,75, p<, 05$, $\left.\eta^{2}=, 01\right]$. La diferencia se produjo en la lectura de ficción $\left(F=2,64, p<, 05, \eta^{2}=, 01\right)$. En la figura 1 puede observarse el incremento de la lectura de ficción en los alumnos del Grado de Maestro en Educación Primaria desde el primer curso al cuarto curso. La media se incrementaba desde 2,43 hasta 2,67.

\section{Figura 1. Lectura de ficción según grado y curso}

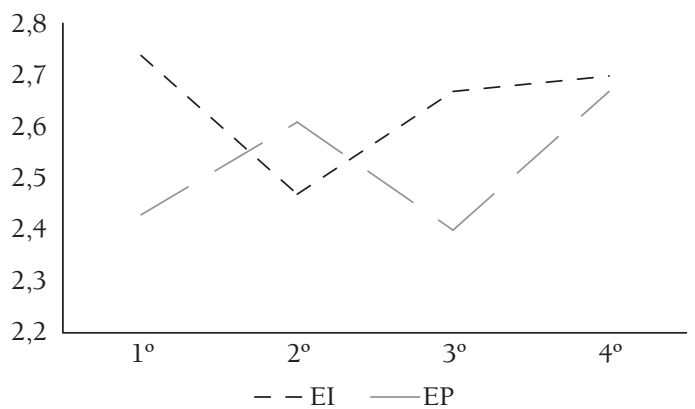

En el análisis del compromiso con la lectura, la interacción del curso y el grado también resultó significativa [Lambda $(5,827)=0,99$, $\left.F=, .40, p<, 05, \eta^{2}=, 02\right]$. La diferencia se produjo en el gusto por la lectura $(F=4,31, p<, 01$, $\left.\eta^{2}=, 01\right)$. En la figura 2 puede observarse el incremento superior del gusto lector en los alumnos del Grado de Maestro en Educación Primaria desde el primer curso al cuarto curso (de $6,42$ a 7,00$)$.

El curso alcanzó significación en el análisis del comportamiento lector [Lambda $(18,2277)=$ $\left.0,92, F=3,82, p<, 001, \eta^{2}=, 03\right]$ y en el compromiso lector [Lambda $(15,2283)=0,95, F=2,72$, $\left.p<, 001, \eta^{2}=, 02\right]$. En la tabla 7 puede observarse el análisis comparativo de las variables que alcanzaron significación.

\section{Figura 2. Gusto lector según grado y curso}

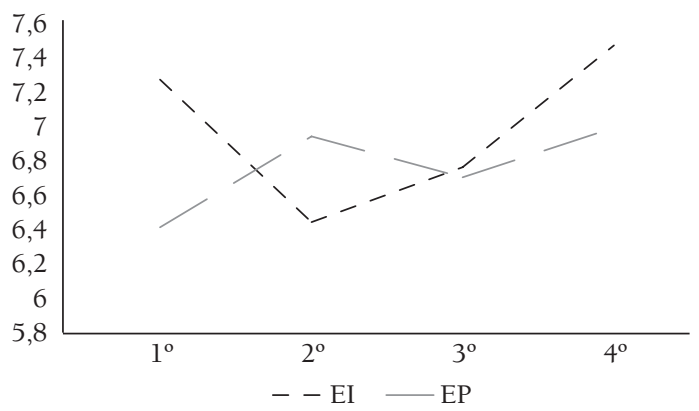


TABla 7. Análisis comparativo de medias según curso

\begin{tabular}{lccccccc} 
& $\mathbf{1}^{\mathbf{0}}$ & $\mathbf{2}^{\mathbf{0}}$ & $\mathbf{3}^{\mathbf{0}}$ & $\mathbf{4}^{\mathbf{0}}$ & $F$ & $\boldsymbol{p}$ & $\eta^{\mathbf{2}}$ \\
\hline Revistas & 1,93 & 2,03 & 2,37 & 2,29 & 10,38 &, 000 &, 04 \\
\hline Motivación extrínseca & 1,36 & 1,42 & 1,64 & 1,77 & 5,31 &, 001 &, 02 \\
\hline $\begin{array}{l}\text { Periodo de lectura } \\
\text { r }\end{array}$ & 1,66 & 1,78 & 1,84 & 1,98 & 2,67 &, 036 &, 01 \\
\hline
\end{tabular}

TABLA 8. Análisis comparativo de medias según grado

\begin{tabular}{lccccc} 
& Infantil & Primaria & $F$ & $p$ & $\eta^{2}$ \\
\hline Motivación intrínseca & & & & & \\
\hline Periodo de lectura & 1,23 & 1,10 & 5,51 &, 019 &, 01 \\
\hline
\end{tabular}

El grado no alcanzó significación en el análisis del comportamiento lector $[$ Lambda $(6,805)=$ $\left.0,99, F=1,72, p=, 113, \eta^{2}=, 01\right]$, pero sí en el compromiso lector [Lambda $(5,827)=0,99, F=$ $\left.2,40, p<, 05, \eta^{2}=, 02\right]$. En la tabla 8 puede observarse el análisis comparativo de las variables que alcanzaron significación.

\section{Conclusiones y discusión}

El primer objetivo de este estudio ha sido analizar la relación del compromiso lector con el hábito lector. Atendiendo a los resultados de investigaciones previas que relacionan el compromiso con la lectura con el rendimiento, con la comprensión y con el progreso lector (Guthrie et al., 2013; Valenzuela et al., 2015; Wigfield et al., 2008), esperábamos encontrar que el compromiso lector se relacionara también con el hábito lector. Los resultados confirman la hipótesis de estudio. Aunque la mayor parte de la varianza del hábito lector viene explicada por el comportamiento lector, el compromiso con la lectura constituye también un componente relevante. La regularidad del comportamiento lector, dedicar parte del tiempo de ocio a la lectura, el gusto por la lectura y estar motivado intrínsecamente son variables que parecen determinantes para tener hábito lector. Investigaciones anteriores ya habían mostrado la importancia de la motivación intrínseca (Applegate y Applegate, 2004) y del gusto por la lectura (Yubero et al., 2014). La consideración del compromiso lector añade a estas variables la relevancia de la regularidad del comportamiento lector y de tener insertada la lectura en el tiempo de ocio. En conclusión, parece relevante la consideración del compromiso lector, tanto para la evaluación como para la intervención sobre los hábitos lectores, ya que aúna elementos que han demostrado su implicación en estudios previos.

Por otra parte, en relación al rol de los maestros como mediadores lectores, el segundo objetivo ha sido analizar el comportamiento y el compromiso lector de los estudiantes de los grados de Maestro en Educación Infantil y Educación Primaria para conocer la evolución competencial como mediadores de lectura en el periodo de formación universitaria. Si, como indican estudios previos (Boggs y Golden, 2009; Cremin et al., 2009; Granado, 2014; Granado y Puig, 2014; Granado et al., 2012), el hábito lector de los educadores y sus experiencias determinan cómo podrán intervenir en su labor de mediadores, es necesario conocer el comportamiento lector y el 
compromiso con la lectura de los maestros. En relación con la formación universitaria, nuestra hipótesis de estudio es que los estudiantes del Grado de Maestro en Educación Primaria, al estar incluido en sus competencias el fomento de la lectura, incrementarán el hábito lector y que, por tanto, se producirá un aumento tanto en el comportamiento lector como en el compromiso con la lectura desde el primer curso hasta su salida de la universidad. Si comparamos los datos sobre el perfil lector previo al acceso a la universidad y el de una vez finalizados los estudios, vemos que se produce una reducción del porcentaje de futuros maestros que se consideran no lectores y se incrementa la percepción de lectores empedernidos. Podría ser que la formación universitaria incidiera más sobre el convencimiento de la necesidad de ser lector como futuro maestro. No obstante, es una medida que puede estar sometida a un elevado impacto del efecto de deseabilidad por mostrar una adecuada imagen profesional de futuro.

En la comparación del hábito lector según el curso que están realizando solamente alcanza significación la evolución en los alumnos del Grado de Maestro en Educación Primaria. Entre el primer curso y el cuarto curso el porcentaje de no lectores se reduce a la mitad y casi se duplica el porcentaje de lectores habituales. En los estudiantes del Grado de Maestro en Educación Infantil la contingencia entre el hábito lector y el curso no alcanza diferencia significativa. Estos resultados suponen un apoyo empírico a la segunda hipótesis de nuestro estudio. Sin embargo, debemos ser prudentes, ya que son alumnos distintos los que se encuentran en cada curso. Para poder confirmar la evolución de los hábitos lectores como consecuencia de los estudios universitarios sería necesario realizar un estudio longitudinal que nos garantizara la evolución personal.

El análisis de las diferencias, atendiendo conjuntamente al curso académico y al grado, muestra la evolución en el comportamiento lector hacia el incremento de la lectura de ficción en los estudiantes del Grado de Maestro en Educación Primaria, y en el componente del compromiso lector centrado en el gusto por la lectura. Estos resultados confirman la hipótesis de estudio, señalando una mejora en los estudiantes del Grado de Maestro en Educación Primaria en los dos componentes evaluados. No obstante, la interacción de las variables muestra una relación diferente por curso según el grado que están cursando, que es difícil de justificar. Sería conveniente llevar a cabo estudios longitudinales para confirmar la relación. También sería interesante realizar estudios cualitativos que aportaran la visión de los alumnos sobre su formación.

El contraste entre los cursos indica diferencias en la tipología lectora y en el compromiso lector en las variables de motivación extrínseca y periodo de lectura. Conforme avanza la formación universitaria, los alumnos leen más revistas, incrementan la motivación extrínseca de la lectura y leen con mayor periodicidad. Estos resultados parecen estar vinculados con la importancia de la lectura como fuente de conocimiento especializado.

Si comparamos en conjunto los hábitos lectores de los futuros maestros con los datos proporcionados por investigaciones previas (Colomer y Munita, 2013; Díaz-Plaja y Prats, 2013; Granado, 2014; Granado et al., 2012; Larrañaga et al., 2008; Sanjuán-Álvarez, 2013), coincidimos en que la mayoría no son lectores habituales. Resulta preocupante que alrededor del 15\% se haya declarado abiertamente no lector. Por otro lado, la categorización del hábito lector indica más de un $20 \%$ de falsos lectores, valor que coincide con el informado en investigaciones previas de estudiantes de Magisterio (Yubero et al., 2014).

Otros estudios han señalado la influencia de las experiencias personales de los maestros sobre la formación lectora de sus alumnos (Boggs y Golden, 2009; Cremin et al., 2009; Granado y Puig, 2015). Los resultados obtenidos en nuestra investigación indican que las experiencias personales 
están constituidas tanto por el comportamiento lector como por el compromiso con la lectura. Por tanto, la universidad, como contexto de formación, debe tener en cuenta ambas dimensiones. Los maestros necesitan una formación completa, sólida y coherente con las competencias que tienen que desarrollar como profesionales. Sin duda, esa tarea es responsabilidad de las universidades y de sus docentes. Para lograr mejores resultados de aprendizaje es necesario incrementar la calidad de la enseñanza, con propuestas que potencien el hábito lector de los futuros maestros, tanto en el comportamiento lector como en el compromiso con la lectura. Sin embargo, como señala Munita (2016), sería reduccionista explicar la construcción de los hábitos lectores de los menores centrándonos exclusivamente en los maestros, ya que son múltiples factores los que intervienen en la construcción lectora.

El interés de esta investigación radica en el estudio del comportamiento y el compromiso lector de los estudiantes del Grado de Maestro en
Educación Infantil y Primaria, ya que son los que, en su futuro profesional, podrán afrontar la formación lectora de niños y niñas. Tal y como defienden Granado y Puig (2014), será necesario promover más actividades de animación a la lectura entre los futuros encargados de llevarlas a cabo en las escuelas, si queremos que sus alumnos lleguen a ser lectores frecuentes y vista la relación que los estudios como PISA (OCDE, 2016) ponen entre hábito y rendimiento, también competentes.

Sería necesario enriquecer este estudio con una investigación longitudinal que nos permita conocer el verdadero papel que juega la universidad sobre los hábitos lectores de los futuros maestros y la relevancia de la formación lectora en aquellos alumnos que decidan cursarla. También sería interesante completar estos datos con una información más intensiva y cualitativa, y ampliar el objeto de estudio a otras poblaciones, como podrían ser los maestros en ejercicio y los futuros profesores de Educación Secundaria.

\section{Referencias bibliográficas}

Applegate, A. J. y Applegate, M. D. (2004). The Peter Effect: Reading habits and attitudes of preservice teachers. The Reading Teacher, 57(6), 554-563. Recuperado de https://thoughtfulliteracy.com/ Applegate\%20and\%20Applegate,\%202004\%20The\%20Peter\%20Effect.pdf

Boggs, M. y Golden, F. (2009). Insights: Literacy memories of preservice teachers self-reported categories of impact. The Reading Matrix, 9(2), 211-223. Recuperado de http://www.readingmatrix. com/articles/sept_2009/boggs_golden.pdf

Cerrillo, P. (2016). El lector literario. México: Fondo de Cultura Económica.

Colomer, T. y Munita, F. (2013). La experiencia lectora de los alumnos de magisterio: nuevos desafíos para la formación docente. Lenguaje y Textos, 38, 37-45. Recuperado de http://www.sedll.org/sites/ default/files/journal/la_experiencia_lectora_de_los_alumnos_de_magisterio._colomer_t.pdf

Comisión Europea (2008). Presidency conclusions. Recuperado de http://www.consilium.europa.eu/ ueDocs/cms_Data/docs/pressData/en/ec/99410.pdf

Cremin, T., Mottram, M., Collins, F., Powell, S. y Safford, K. (2009). Teachers as readers: building communities of readers. Literacy, 43, 11-19. doi:10.1111/j.1754-8845.2007.00001.x

De-Naeghel, J., Valcke, M., De-Meyer, I., Warlop, N., Van-Braak, J. y Van-Keer, H. (2014). The role of teacher behavior in adolescents' intrinsic Reading motivation. Reading and Writing, 27(9), 1547 1565. doi: 10.1007/s11145-014-9506-3

Díaz-Plaja, A. y Prats, M. (2013). Recordar, aprender, practicar: qué sabe y qué debe saber un futuro maestro. Lenguaje y Textos, 38, 19-28. Recuperado de http://www.sedll.org/sites/default/files/ 
journal/recordar_aprender_practicar_que_sabe_y_que_debe_saber_un_futuro_maestro._diazplaja_a._y_ot.pdf

Eurydice (2011). La enseñanza de la lectura en Europa: contextos, políticas y prácticas. Madrid: Ministerio de Educación. doi: 10.2797/63712

Felipe, A. (2016). Competencia, estrategias y hábitos lectores de maestros en formación inicial (tesis doctoral). Málaga: Universidad de Málaga.

Granado, C. (2014). El docente como lector: un estudio de los hábitos lectores de futuros docentes. Cultura y Educación, 26(1), 44-70. doi: 10.1080/11356405.2014.908666

Granado, C. y Puig, M. (2014). ¿Qué leen los futuros maestros y maestras? Un estudio del docente como sujeto lector a través de los títulos que evocan. Ocnos, 11, 93-112. doi: 10.18239/ ocnos_2014.11.05

Granado, C. y Puig, M. (2015). La identidad lectora de los maestros en formación como componente de su identidad docente. Un estudio de sus autobiografías como lectores. Ocnos, 13, 43-63. doi: 10.18239/ocnos_2015.13.03

Granado, C., Puig, M. y Romero, C. (2012). Los futuros maestros y maestras ante la educación lectora: hábitos lectores, creencias, capacitación adquirida y necesidades formativas de los futuros maestros y maestras respecto a la educación lectora. Sevilla: Consejería de Cultura de Andalucía. Recuperado de http://www.juntadeandalucia.es/cultura/web/html/sites/consejeria/areas/letras/ Galerias/Adjuntos/estudios/3_Estudio_Futuros_maestros_y_maestras_ante_la_educacixn_ectora.pdf

Guthrie, J. T., Klauda, S. L. y Ho, A. N. (2013). Modeling the Relationships Among Reading Instruction, Motivation, Engagement, and Achievement for Adolescents. Reading Research Quarterly, 48(1), 9-26. doi:10.1002/rrq.035

Guthrie, J. T., Wigfield, A., Metsala, J. L. y Cox, K. E. (1999). Cognitive and motivational predictors of text comprehension and reading amount. Scientific Studies of Reading, 3, 231-236.

Guthrie, J. T. y Wigfield, A. (2000). Engagement and motivation in reading. En M. L. Kamil, P. B. Mosenthal, P. D. Pearson y R. Barr (eds.), Handbook of reading research ( $3^{\text {rd }}$ ed., pp. 403-422). New York: Longman.

López-Valero, A., Encabo, E. y Jerez, I. (2016). Consideraciones docentes e investigadoras para la didáctica de la literatura en Educación Infantil y en Educación Primaria. Lenguaje y Textos, 43, 7-14. doi: $10.4995 /$ lyt.2016.5820

Ministerio de Educación (2007). PIRLS 2006. Estudio internacional de progreso en comprensión lectora de la IEA. Madrid: Instituto Nacional de Evaluación Educativa. Recuperado de https://www. mecd.gob.es/inee/evaluaciones-internacionales/pirls/pirls-2006.html

Munita, F. (2016). Prácticas didácticas, creencias y hábitos lectores del profesor en una escuela exitosa en la promoción lectora. Ocnos, 15(2), 77-97.doi: 10.18239/ocnos_2016.15.2.1140

Organización para la Cooperación y el Desarrollo Económicos (2016). Resultados clave. Recuperado de https://www.oecd.org/pisa/pisa-2015-results-in-focus-ESP.pdf

Torres, A. y Vallejo, M. (2018). ¿Contribuye la universidad al desarrollo de aprendizaje de calidad? Estudio descriptivo con estudiantes de la Universidad de Murcia. Revista Interuniversitaria de Formación del Profesorado, 21(1), 129-142. doi: 10.6018/reifop.21.1.295261

Valenzuela, J. P., Gómez, G. y Sotomayor, C. (2015). The role of reading engagement in improving National Achievement: An análisis of Chile's 2000-2009 PISA results. International Journal of Educational Development, 40, 28-39. doi: 10.1016/j.ijedudev.2014-11-011

Wigfield, A., Guthrie, J. T., Perencevich, K. C., Taboada, A., Klauda, S. L., McRae, A. y Barbosa, P. (2008). Role of reading engagement in mediating effects of reading comprehension instruction on readig outcomes. Psychology in the Schools, 45(5), 432-445. doi: 10.1002/pits.20307 
Yubero, S. y Larrañaga, E. (2015). Lectura y universidad: estudio de los hábitos lectores de los estudiantes universitarios de España y Portugal. El Profesional de la Información, 24(6), 713-719. doi: 10.3145/epi.2015.nov.03

Yubero, S., Larrañaga, E. y Pires, N. (2014). Estudo sobre os hábitos de leitura dos estudantes protugueses do ensino superior. Castelo Branco, Portugal: Instituto Politécnico de Castelo Branco. Recuperado de http://pnl2027.gov.pt/np4/file/198/Estudo_Alunos_Ens_Superior.pdf

\section{Abstract}

The complex relationship of teachers with reading as a skill: the reading behavior of the teachers of Pre-school and Primary Education in training

INTRODUCTION. The interest of this work is to reflect, specifically, on the quality of training of future teachers in the specific competence field as mediators of reading. On the one hand, the latest reports on the reading habits of Spanish students call into question that there has been evolution in this matter, so it seems necessary to make more effective the teaching of the reading skill at the first stages of education. On the other hand, previous research has pointed out the influence exerted by teachers' reading experiences on their effectiveness in this field. This research is aimed at studying the behavior and the reading engagement of the students of the Teachers' Degree to reflect on their competence development regarding the reading education of the children of Pre-school and Primary Education. METHOD. This research has involved 861 students from the Universities of Castilla-La Mancha and Santiago de Compostela, from the first to fourth year of the Teachers' Degree in Pre-school Education (355) and Primary Education (506). Reading behavior and engagement to reading were evaluated. RESULTS. The results indicate the relationship between behavior, engagement and reading habits. As they advance in the training, there is an increase in the reading habits in the students of the Teachers' Degree in Primary Education, but not in the ones of Pre-school Education. The category of false readers is evident, as well as a high percentage of future teachers who do not seem to have the necessary personal conditions to develop a voluntary reader behavior in their students. DISCUSSION. The university must move towards a complete, solid and coherent training with the competences that these future professionals have to develop as mediators of reading, strengthening their reading habits as regards both to behavior and reading engagement.

Keywords: Reading Habits, Reading Interests, Teachers, Reading Teachers, University.

\section{Résumé}

La complexe relation des enseignants avec la lecture: le comportement de lecture des enseignants de l'éducation maternelle et primaire en formation

INTRODUCTION. Lintérêt de ce travail est de réfléchir, en particulier, sur la qualité de la formation des futurs enseignants dans le domaine spécifique qui les compète en tant que médiateurs de la lecture. Les derniers rapports des habitudes de lecture des élèves espagnols questionnent qu'il y ait une évolution dans cette matière, il semble donc nécessaire de rendre l'enseignement des premiers stades éducatifs plus efficace. D'autre part, des recherches antérieures 
ont souligné l'influence exercée par les expériences de lecture des enseignants sur leur efficacité dans ce domaine. Cette recherche vise à étudier le comportement et l'engagement en lecture des étudiants universitaires du Degré d'Enseignant à réfléchir sur leur compétence en formation à la lecture des enfants des niveaux maternelle et primaire. MÉTHODE. Dans cette recherche ont participé 861 étudiants des universités de Castilla-La Mancha et de Santiago de Compostela, de la première au quatrième cours du Degré d'Enseignant de l'Éducation Maternelle (355) et l'Éducation Primaire (506). Le comportement des lecteurs et l'engagement à la lecture ont été évalués. RÉSULTATS. Les résultats ont indiqué la relation entre le comportement, l'engagement et les habitudes de lecture. Pendant que les étudiants progressent dans sa formation, il se produit une augmentation de l'habitude de lecture dans les étudiants du Degré d'Enseignant du niveau Primaire, mais pas dans les cas des étudiants du Degré d'Enseignant de Maternelle. Il a aussi montré la catégorie de faux lecteurs, ainsi qu'un pourcentage élevé de futurs enseignants qui semblent ne pas répondre aux conditions personnelles précises pour le développement d'un comportement lecteur volontaire dans leurs élèves. DISCUSSION. Luniversité doit aller vers une formation complète, solide et cohérente avec les compétences que les futurs professionnels doivent développer en tant que médiateurs de la lecture, en améliorant leurs habitudes de lecture en ce qui concerne à la fois le comportement et l'engagement en lecture.

Mots-clés: Habitudes des Lecteurs, Intérêt par la Lecture, Enseignants, Professeurs Lecteurs, Université.

\section{Perfil profesional de los autores}

\section{María Elche Larrañaga (autora de contacto)}

Doctora en Investigación en Humanidades, Artes y Educación por la Universidad de Castilla-La Mancha (UCLM). Es personal investigador de la UCLM y colaboradora del Centro de Estudios de Promoción de la Lectura y la Literatura Infantil (CEPLI), en el grupo de investigación "Psicología, Educación y Lectura" (PEL) y la revista Ocnos.

ORCID: http://orcid.org/0000-0002-7148-7958

Correo electrónico de contacto: maria.elche@uclm.es

Dirección para la correspondencia: Universidad de Castilla-La Mancha, Facultad de Ciencias de la Educación y Humanidades. Avenida de los Alfares, 42. 16071 Cuenca, España.

\section{Santiago Yubero Jiménez}

Doctor en Psicología y licenciado en Pedagogía, es catedrático de la Escuela Universitaria de Psicología Social de la Educación. Es decano de la Facultad de Ciencias de la Educación y Humanidades de Cuenca (UCLM), director del Centro de Estudios para la Promoción de la Lectura y Literatura Infantil (CEPLI), de la Universidad de Castilla-La Mancha, codirector del Máster de Promoción de la Lectura y Literatura Infantil y director de la revista Ocnos. Coordina el grupo de investigación "Psicología, Educación y Lectura" (PEL).

ORCID: http://orcid.org/0000-0002-7148-7958

Correo electrónico de contacto: santiago.yubero@uclm.es 